\title{
Contextual Awareness as Measure of Human- Information Interaction in Usability and Design
}

\author{
Michael J. Albers \\ East Carolina University \\ Greenville NC 27858, USA \\ albersmaecu.edu
}

\begin{abstract}
Contextual awareness (CA) [3] provides a way of thinking about the communication quality of the human-information interaction (HII) aspects of a design [4]. Understanding information in complex situations is essentially always cognitively-based rather than physically-based (although physical interaction may be required to control the situation). Gaining that understanding within a complex situation requires mentally integrating many pieces of information, which requires the user knows the information exists, what it means, and how it is interrelated to other pieces of information. The design and testing of complex information systems and effectively interacting with them require a different approach than working with simply information $[1,11]$.
\end{abstract}

Keywords: contextual awareness, human-information interaction, usability.

\section{Definition of Contextual Awareness}

Contextual awareness (CA) is the understanding of the information within an informational situation which forms the basis for how to interpret new information and how to make decisions for interacting with that situation. CA is about a person's state of knowledge about the situation and not about the process they use to obtain that knowledge. With poor CA, people can know something is occurring or that a particular piece of information exists, but they cannot easily find relevant information or they have the information but do not understand how it relates to the overall situation. On the other hand, good CA does not guarantee a person will form the proper intention or make the proper decisions; the error analysis literature is filled with cases where people understood a situation, but still made incorrect choices.

Situations which require design teams to consider CA are those which contain complex information [2] that must fit within the situation and be presented to meet a reader's information needs. Understanding a situation requires mentally integrating many pieces of information with respect to the reader's goals and the current context. In complex situations, people don't follow a fixed path, instead they continually adjust their mental paths as new information presents itself. The non-fixed path arises from the non-linear aspects of problem solving. Addressing the mental shift to non-linear thinking breaks with the fundamental philosophy of step-by-step analysis. The design must place the information within the situational context and allow user to develop and maintain an overall awareness of the situation. 
CA provides a tool to help judge the fluid, ill-formed goals in complex situations. CA pulls back from thinking in terms of button pushing or fonts/color choices. Instead, it focuses on content and relationships across screens with a focus on how to efficiency and effectively communicate information. Different people will use different methods and approaches to gain contextual awareness.

Elements of good contextual awareness are (figure 1): (1) Understands how the information fits within the current situation. (2) Understands the information relationships [1]. Information comprehension requires knowing how information relates to other information. (3) Understands the future development of the situation and can make predictions about the ripple effect of different decisions.

\section{Why Be Concerned about Contextual Awareness}

Design teams need to consider how people need the information to develop contextual awareness.. It is not enough to simply provide them with the information; it must be organized and presented in a way that lets readers fit that information into their background knowledge and build up a coherent understanding of the entire situation.

If people are to create a coherent story, they need information to enable them to comprehend the situation. They can use that comprehension as the basis for the selection and performance of judgments, decisions, and actions. Gaining that understanding requires mentally integrating many pieces of information, which requires the person knows the information exists, what it means, and how it is interrelated to other pieces of information. That implies having a deep enough understanding to understand the current situation and to be able to make predictions about its future evolution.

Having full CA in a complex situation typically requires more than just possessing the available information; in addition, environmental and social aspects must also be considered. Schriver [12] discusses the importance of the social situation and how failing to allow for its effects leads to failure of information communication.

\subsection{Information Comprehension}

People identify a problem and make a problem-solving decision based on how they interpret the information around them. Unfortunately, the interpretation often has less to do with reality and more to do with what the person expects to see. Strong expectancy biases in data interpretation must be expected [8]. Also, the presentation format exerts a strong influence on how people interpret the data. Salient information must have proper presentation. With poor information salience, the presentation fails to either effectively present salient information or to present adequate cues about relevant information.

Much of the information analysis research attempts to predefine people's needs and, thus, the system breaks down when users go beyond the solution envisioned by the designer. User analysis and task analysis are important components of current design methodology, but are not constructed to support complex problem solving, rather they focus on assembling task blocks versus defining the continuous chain of event that exist in a problem solving situation. In a well-defined domain, the user 


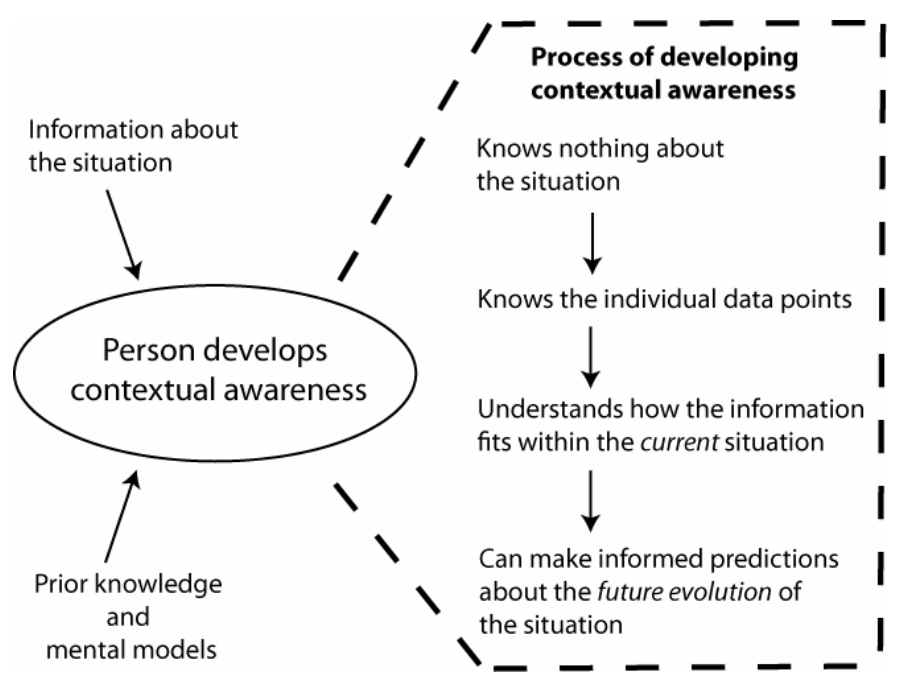

Fig. 1. Developing contextual awareness

approaches are limited and can be fully defined by the designer. In an ill-structured domain, each user takes a slightly different path and the designer can't assume that an understanding of how one person performs the task describes anyone else.

\subsection{Information Relationships}

Effective design for $\mathrm{CA}$ requires focusing on the relationships between information and how those relationships fit into the user's mental model of the situation. As people address a complex problem, a structure emerges which is based on the context, the relationships between the important elements, and the user's mental model. When solving a problem, the user's initial difficulty is recognizing what is wrong and what information is needed to verify the problem and suggest possible solutions.

Multiple studies have on how people solve problems in the real-world reveal a consistent feature of people constantly adjusting their goals and sub-goals to allow for the dynamic nature and quality of the information available [9, 10]. Unfortunately, people have a hard time integrating information and relating various data points to each other. Unless it is properly organized, humans cannot efficiently process large amounts of information, especially with the incomplete information available in a typical real-world context [7, 13].

\subsection{Evolution of the Situation's Future}

After understanding the relationships with the situation, the person needs to use the data and relationships to make predictions of the results of potential decisions. Complex situations are highly dynamic and usually exhibit a non-linear response to changes. But people make linear extrapolations and are extremely poor at judging non-linear data. Many failures occur because of the assumption that the initial conditions would somehow suddenly jump from initial to final state. 


\section{How to Analyze and Design for Contextual Awareness}

Because, by definition, CA is about people understanding the situation and being able to make predictions of the situation's future development, it leads directly to methods which can be used during early prototyping and usability testing of reading to decide information. Note that this is substantially different from a more typical usability test which focuses on how people obtain information or perform a task (e.g., measuring search times or how paths deviate from optimal). Instead, it tests a person's comprehension of the overall situation and the information presented.

The intial analysis reveals the general user goals and information needs and the potential sub-goals that must be addressed as they proceed to a resolution. Defining the goals and information needs becomes complicated because there is no "right way" to solve a complex problem $[2,10]$. With complex situations, there is no single path and no way of predicting when the user will make a decision. Slightly different starting points can require different information and resulting decisions.

The major problem in decision making is understanding the problem. A decision is based on how people interpret the information around them. Incorrect or incomplete information can lead to incomplete or invalid decisions. Even with complete information, different presentations cause different approaches to the solution [6]. Having the data available does not equal understanding. Thus, the users' main need is information which supports gaining a clear understanding of the problem and the possible solutions. During the requirements analysis, the information designer must gain an understanding of what information is relevant, how the information is obtained, how it relates to other information, and how to present the information to maximize revealing those relationships.

The first step in designing an effective system should not be to define the data needs, but to define the communication situation in terms of the necessary information processes and mental models of the users. A standard practice for handling complexity is to attempt to limit the problem-space and accept that all the information needed can be defined in advance [5]. Rather than attempt to handle the entire dynamic process, this type of analysis focuses on single slices (either time or procedure). It often results in ignoring parts or misestimating each part's importance to understanding the overall situation [13]. Many methods attempt to define a static space and attempts to predefine the space and, thus, the system breaks down when users try to go beyond the solution envisioned by the designer.

The situation analysis for determining a person's contextual awareness must be performed in the context it is designed to address. An underlying problem facing the analyst is that there is no simple way of determining when to stop the user analysis, but it does run the risk of trying to collect everything. Techniques such as critical incident analysis can provide a relatively bounded area of interest in which to collect user goals and information needs which can be directly related back to the situation. The base situation analysis must get into the person's mind and start to understand the mental model building and situational thought processes while it builds a picture of how the person becomes fully aware of the overall situation. 


\section{Conclusion}

Effective design for complex problem-solving focuses on providing the appropriate content for the user's real-world goals and information needs. The design must not be swept up in the technology and, instead, must focus on how the information and presentation apply to the situational context. CA adds to the HCI and usability testing toolbox for creating and testing information designed to address open-ended questions for problem-solving or decision-making.

\section{References}

1. Albers, M., Still, B. (eds.): Usability of Complex Information Systems: Evaluation of User Interaction. CRC Press, Boca Raton (2010)

2. Albers, M.: Communication of complex information: User goals and information needs for dynamic web information. Erlbaum, Mahwah (2004)

3. Albers, M.: Design for Effective support of user intentions in information-rich interactions. Journal of Technical Writing and Communication 39(2), 177-194 (2009)

4. Albers, M.: Human-Information Interaction and Technical Communication: Concepts and Frameworks. IGI Global (in press)

5. Basden, A., Hibberd, P.: User interface issues raised. by knowledge refinement. International Journal of Human-Computer Studies 45, 135-155 (1996)

6. Elam, J., Mead, M.: Can software influence creativity? Information Systems Research 1(1), 1-10 (1990)

7. Gerlach, J., Kuo, F.: Understanding human-computer interaction for information system design. MIS Quarterly 15(4), 527-550 (1991)

8. Klein, G.: Do decision biases explain too much. Human Factors Society Bulletin 32(5), 1-3 (1988)

9. Klein, G.: Sources of Power: How People make Decisions. MIT Press, Cambridge (1999)

10. Mirel, B.: Applied constructivism for user documentation. Journal of Business and Technical Communication 12(1), 7-49 (1998)

11. Redish, J.: Expanding usability testing to evaluate complex systems. Journal of Usability Studies 2(3), 102-111 (2007)

12. Schriver, K.: Dynamics in Document Design. Wiley, New York (1996)

13. Woods, D., Roth, E.: Cognitive engineering: Human problem solving with tools. Human Factors 30(4), 415-430 (1988) 